\section{Bringing more benign materials into the built environment}

\section{By Sara Tepfer}

W e spend $90 \%$ of our time indoors, which means that the design of our built environments has the potential to greatly affect our health and well-being. Architectural design for human health can be traced back for many generations, but only recently have questions surrounding design and human health been extended to the material and chemical scales. Driven by a widespread interest in "healthy buildings" and the mitigation of the many health hazards posed by chemicals in building materials, architects are increasingly interested in the chemistries of the materials they use.

This new interest requires collaboration and technical knowledge transfer from chemistry, materials science, and environmental health science to architecture. Historically, collaboration among these many disciplines has mostly been driven by regulation (e.g., asbestos abatement). More recently, however, the dialogue is shifting from the regulatory to the market sphere, and with this shift has come a host of design guidance, green building certification criteria, and collective knowledge growth around the challenges and opportunities at the interface of materials science, environmental health science, and architecture.

Arup* and the American Institute of Architects recently published a guidance

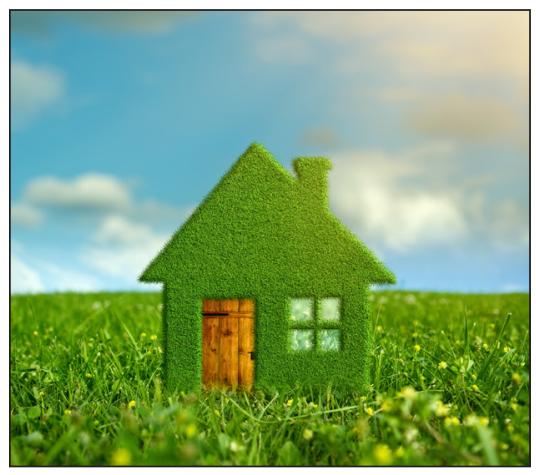

report examining this intersection. The Prescription for Healthier Building Materials: A Design and Implementation Protocol presents an introductory review of the knowledge around healthier building materials and the opportunities and challenges in translating this knowledge to architectural practice. This article presents the findings of that report that its authors deem most relevant to the materials science community.

Awareness: Awareness is essential to create change. In this case, it's the awareness of the challenges associated with developing and transitioning to healthier building materials. Among the many challenges is the lack of publicly available data: The majority of industrially available chemicals go unregulated, and, as such, there is limited hazard and exposure data available. To enable action in the face of such data limitations, the building industry's transition to healthier materials relies on the precautionary principle. The principle states that we should refrain from using chemicals whose ultimate effects are disputed or unknown.

Fluency: The design community cannot be experts in chemical risk assessment, though that is the current expectation. This is avoidable, and chemists and materials scientists can help. The more chemists and materials scientists understand and consider health and environmental impacts during the design of both chemical processes and products, there is less need to mitigate them later. In turn, this reduces the architects' need to assess and avoid hazardous materials in the design of buildings. A fundamental understanding of chemical hazard and risk is ess ential during the development of new chemical syntheses, and materials are essential to make the changes the building industry needs.
Innovation: Design innovations are essential across the chemical, material, and architectural scales. However, the innovation cycle for reformulation does not typically align with the pace of an architectural design project-innovation across these disciplines operates at different time scales. As such, architects are "designing out" hazards at the building scale through alternative building- and product-scale solutions, rather than asking for new chemical processes and formulations. Because designing out hazards at the architectural scale is not always possible, innovations in both chemistry and materials science are needed.

Transparency: These innovations can be motivated by increased transparency around chemicals and chemical hazards. With more transparency comes greater potential for change. Greater materials selection scrutiny is applied when chemical composition and hazard data are made publicly available. This transparency enables educated decision making and, in turn, triggers innovation away from problem chemicals. This innovation leads to safer materials on the market. We can inspire innovation toward safer chemistries of building materials through transparency around chemical composition.

Education: Materials science education has a role to play in this transition to more benign building materials. We can train chemistry and materials science students to approach the design of process and product with an eye toward hazard reduction. While regulation remains slow, market drivers are increasing the value of greener chemistries and creating a demand for chemists with training in that area.

This is an exciting time for anyone interested in the relationships among these disciplines. Identifying solutions that bring more benign materials into the built environment has huge implications for human health. This space houses a huge invitation for greener and more sustainable chemistry and materials science. The materials science community has an opportunity to contribute to our progress toward healthier built environments. 